\title{
Ultrahigh-sensitive optical coherence elastography
}

\author{
Yan Li (10 ${ }^{1,2}$, Sucbei Moon ${ }^{1,3}$, Jason J. Chen (1) ${ }^{1,2}$, Zhikai Zhu ${ }^{1,2}$ and Zhongping Chen ${ }^{1,2}$
}

\begin{abstract}
The phase stability of an optical coherence elastography (OCE) system is the key determining factor for achieving a precise elasticity measurement, and it can be affected by the signal-to-noise ratio (SNR), timing jitters in the signal acquisition process, and fluctuations in the optical path difference (OPD) between the sample and reference arms. In this study, we developed an OCE system based on swept-source optical coherence tomography (SS-OCT) with a common-path configuration (SS-OCE $\mathrm{CP}_{\mathrm{P}}$ ). Our system has a phase stability of $4.2 \mathrm{mrad}$ without external stabilization or extensive post-processing, such as averaging. This phase stability allows us to detect a displacement as small as $\sim 300 \mathrm{pm}$. A common-path interferometer was incorporated by integrating a 3-mm wedged window into the SS-OCT system to provide intrinsic compensation for polarization and dispersion mismatch, as well as to minimize phase fluctuations caused by the OPD variation. The wedged window generates two reference signals that produce two OCT images, allowing for averaging to improve the SNR. Furthermore, the electrical components are optimized to minimize the timing jitters and prevent edge collisions by adjusting the delays between the trigger, k-clock, and signal, utilizing a high-speed waveform digitizer, and incorporating a high-bandwidth balanced photodetector. We validated the SS$\mathrm{OCE}_{\mathrm{CP}}$ performance in a tissue-mimicking phantom and an in vivo rabbit model, and the results demonstrated a significantly improved phase stability compared to that of the conventional SS-OCE. To the best of our knowledge, we demonstrated the first SS-OCE $\mathrm{CP}_{\text {s }}$ ystem, which possesses high-phase stability and can be utilized to significantly improve the sensitivity of elastography.
\end{abstract}

\section{Introduction}

Optical coherence elastography (OCE) is an emerging functional imaging technique that quantifies the elasticity of biological tissue by using Doppler optical coherence tomography $(\mathrm{OCT})$ to measure the local tissue displacement as a function of the applied stress ${ }^{1,2}$. Compared with conventional elastography (e.g., magnetic resonance elastography, ultrasound elastography, and Brillouin microscopy), OCE possesses micron-level resolution and axial displacement sensitivity on the order of subnanometres and therefore has become an attractive research tool for ophthalmology, dermatology, cardiology, and oncology $^{3-9}$.

\footnotetext{
Correspondence: Zhongping Chen (z2chen@uci.edu)

${ }^{1}$ Beckman Laser Institute, University of California, Irvine, Irvine, CA 92612, USA

${ }^{2}$ Department of Biomedical Engineering, University of California, Irvine, Irvine, CA 92617, USA

Full list of author information is available at the end of the article.
}

Since OCE relies on the measuring phase via Doppler $\mathrm{OCT}$, the phase stability of the imaging system is the key factor determining its performance ${ }^{10-12}$. Most OCE systems utilizing the acoustic radiation force (ARF) as a tissue excitation method are reported to have the capability of detecting displacements in the range of hundreds of nanometers. In those cases, a relatively strong ARF is necessary to accurately reconstruct the elastic wave propagation, and this force may exceed the ophthalmic mechanical index (MI) safety standard of 0.23 approved by the Food and Drug Administration ${ }^{13-15}$. An OCE system with ultrahigh displacement sensitivity will be able to scale down the applied ARF by at least 1 order of magnitude while maintaining a sufficient signal-tonoise ratio (SNR), which will reduce the required ARF such that it is within the range of the MI safety standard to facilitate the clinical translation of OCE in ophthalmology. The common-path configuration, as an optical method, addresses the phase instability caused by

\section{(c) The Author(s) 2020}

\footnotetext{
(c) Open Access This article is licensed under a Creative Commons Attribution 4.0 International License, which permits use, sharing, adaptation, distribution and reproduction in any medium or format, as long as you give appropriate credit to the original author(s) and the source, provide a link to the Creative Commons license, and indicate if changes were made. The images or other third party material in this article are included in the article's Creative Commons license, unless indicated otherwise in a credit line to the material. If material is not included in the article's Creative Commons license and your intended use is not permitted by statutory regulation or exceeds the permitted use, you will need to obtain permission directly from the copyright holder. To view a copy of this license, visit http://creativecommons.org/licenses/by/4.0/.
} 
environmental vibration that leads to a fluctuating optical path difference (OPD) between the reference and sample arms. Additionally, the common-path configuration provides intrinsic compensation for polarization mismatch and dispersion mismatch between the sample and reference arms ${ }^{16-18}$. In 2017, Lan et al. reported a high-phasestability OCE system using a common-path spectraldomain OCT (SD-OCT) to achieve a subnanometre displacement sensitivity, demonstrating the feasibility of common-path OCE; however, only phantom experiments were performed $^{19}$. In addition, SD-OCT-based OCE systems are based on a static operation principle that provides phase-stable detection ${ }^{15,20-24}$, but their performance is limited by the groove density of the diffraction grating, the center wavelength of the light source, and the resolution of the line-scan camera, hence the inherent disadvantages of phase washout, low imaging speed, shallow penetration depth, and short imaging range ${ }^{25,26}$.

In contrast, swept-source optical coherence tomography (SS-OCT), which can be operated with a much narrower instantaneous linewidth, longer center wavelength, higher repetition rate, and balanced detection, has the capability of providing a long imaging range, deep penetration depth, high imaging speed, and reduced fringe washout. Nevertheless, the phase stability of SS-OCT suffers from fluctuations in the mechanical movement of the sweeping laser; thus, a proper synchronization between the data acquisition and laser sweep is crucial in achieving a highphase stability in a swept-source system ${ }^{27}$. A lambda $(\lambda)$ trigger using a fiber Bragg grating (FBG) as well as a kclock generated by a Mach-Zehnder interferometer (MZI) have been introduced to improve the phase stability, making SS-OCT a more attractive setup in OCE applications $^{28}$. In recent years, several OCE systems based on conventional SS-OCT (SS-OCE $\mathrm{COV}_{\mathrm{C}}$ ) have been proposed $^{14,29-35}$, and their feasibilities have been validated through ex vivo and in vivo experiments, demonstrating great potential towards clinical translation. Although these studies have reported subnanometre displacement sensitivities, this is achieved only in system charaterization where a simple common-path configuration is used.

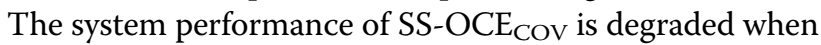
performing measurements because the phase fluctuation between the sample and reference arms is not negligible in conventional OCE. Furthermore, the reported SNR and phase stability are usually from the averages of several measurements ${ }^{36}$. Despite current advancements in SSOCT, achieving a phase sensitivity on the order of subnanometres from actual experiments using SS-OCE remains challenging.

In our study, we designed and implemented a highphase-stable OCE system using a common-path SS-OCT $\left(\mathrm{SS}-\mathrm{OCE}_{\mathrm{CP}}\right)$. A 3-mm thick, 30-arcmin wedged glass window (WW10530, Thorlabs, Inc., NJ) was incorporated distal to the scan lens to generate two reference signals, each from one of the surfaces of the window. This setup allows for the simultaneous generation of two OCT interference signals in two different frequency domains, which can be averaged for an enhanced SNR and reduced speckle. This averaging method is not achievable in SDOCT due to the limited imaging range. Furthermore, the common-path configuration minimizes the differences between the sample and the reference arm, thereby providing stable phase information for precision displacement measurement. In addition to the optical method, data acquisition and synchronization were optimized to accurately retrieve the phase information. In this report, we first compared the phase performance of SS-OCE $E_{C P}$ with that of $\mathrm{SS}-\mathrm{OCE}_{\mathrm{COV}}$. Then, a tissue-mimicking phantom model and an in vivo rabbit model were imaged to validate the $\mathrm{SS}-\mathrm{OCE} \mathrm{E}_{\mathrm{CP}}$ performance.

\section{Results}

\section{Phase stability quantification}

To measure the phase stability of our SS-OCE $\mathrm{CP}_{\mathrm{CP}}$, a 1.0$\mathrm{mm}$ microscope slide was placed at the focus of the objective lens to generate autocorrelation interference by the back-reflected light from the front and back surfaces of the slide. In our SS-OCE $\mathrm{COV}_{\mathrm{C}}$ counterpart, a gold mirror was placed at the focus of the objective lens to generate an OCT interference signal with the same frequency as that of SS-OCE $E_{C P}$. The length of the reference arm was adjusted by $1.40 \mathrm{~mm}$ to offset the zero OPD. In both cases, 5000 A-lines were acquired sequentially. Figure $1 \mathrm{a}, \mathrm{b}$ shows the overlaid interference fringes $(n=5000)$ from SS-OCE $\mathrm{COV}$ and SS-OCE $\mathrm{CP}_{\mathrm{CP}}$, respectively. Temporal shifting is much more severe in SS-OCE $\mathrm{COV}_{\text {, }}$ which implies a better timing stability in SS-OCE $\mathrm{CP}_{\mathrm{CP}}$. For the temporal analysis, the timing information of each zero-crossing was obtained through linear interpolation (blue and red dashed boxes in Fig. 1a, b, respectively), and the standard deviation of the timing variation corresponds to the phase stability of the system. For SS$\mathrm{OCE}_{\mathrm{COV}}$, the standard deviation was calculated to be $940 \mathrm{ps}$, whereas that of SS-OCE $\mathrm{CP}_{\mathrm{C}}$ was $35 \mathrm{ps}$. The corresponding histograms are shown in Fig. 1c. In addition, the phase stabilities of SS-OCE $\mathrm{COV}_{\text {and }}$ SS-OCE $\mathrm{CP}_{\mathrm{CP}}$, which were $175 \mathrm{mrad}$ ( $13-\mathrm{nm}$ displacement) and $4.2 \mathrm{mrad}$ ( 0.3-nm displacement), respectively, were determined by calculating the standard deviation of the phase angle at the peak in the frequency domain, demonstrating a $>40$ fold phase stability improvement in SS-OCE $E_{\mathrm{CP}}$ compared to SS-OCE $\mathrm{COV}_{\text {. }}$

\section{Enhanced SNR}

Since the 3-mm wedged window generates two OCT images in different frequency domains, these two OCT images can be averaged to enhance the SNR, further 

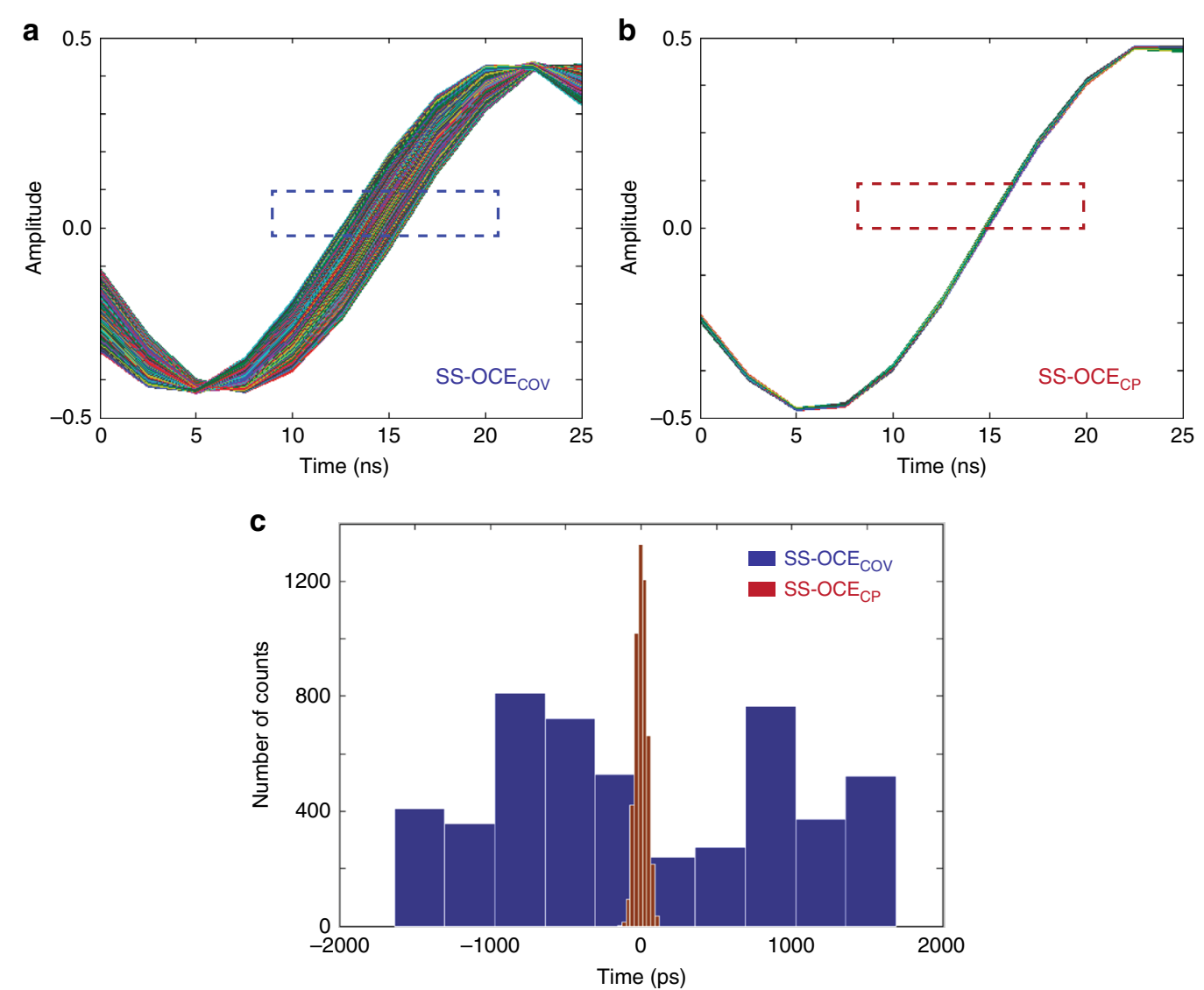

Fig. 1 Phase stability quantification. Overlay of 5000 interference fringes obtained using (a) SS-OCE zero crossings

improving the phase stability. Figure 2a shows the OCT images of the phantom obtained via $\mathrm{SS}-\mathrm{OCE}_{\mathrm{CP}}$; the top and bottom images are the interference signals generated by the back-reflected light from the back and front window surfaces, respectively, with the backscattered light from the phantom. The high-intensity horizontal line between the two OCT images is the interference signal from the two surfaces of the window. The two images are separated by $\sim 3.4 \mathrm{~mm}$. By averaging the two images, the SNR was improved from 73 and 71 to $76 \mathrm{~dB}$, an approximately $3-\mathrm{dB}$ improvement (Fig. $2 \mathrm{~b}$ ). The enhanced SNR through averaging is also reflected on the Doppler OCT images. Figure 2c shows the pair of Doppler images obtained via SS-OCE $\mathrm{CP}_{\mathrm{CP}}$, and Fig. $2 \mathrm{~d}$ shows the averaged result, demonstrating the reduced noise floor and hence improved SNR.

\section{Phantom experiment}

After demonstrating the improved SNR and phase stability of the SS-OCE $\mathrm{CP}_{\mathrm{CP}}$ system, the elastograms of the phantom obtained using both SS-OCE $\mathrm{COV}_{\text {and }}$ SS$\mathrm{OCE}_{\mathrm{CP}}$ were compared. The time-lapse Doppler images of SS-OCE $\mathrm{COV}_{\mathrm{CO}}$ and $\mathrm{SS}-\mathrm{OCE} \mathrm{EP}_{\mathrm{CP}}$ are shown in Fig. $3 \mathrm{a}-\mathrm{d}$ and Fig. $3 \mathrm{e}-\mathrm{h}$, respectively. While an outward propagation of the spherical elastic waves was observed in both cases, the SS-OCE $\mathrm{CP}_{\mathrm{CP}}$ result reveals a more pronounced boundary between the upward (yellow) and downward (blue) displacement, denoted by the white * in Fig. 3. In addition, this displacement boundary is better maintained in the deeper region when imaged using SS-OCE $\mathrm{CP}_{\text {(same white }}$ " in Fig. 3b, f). Since the deeper region of the image is encoded in the higherfrequency components of the interference signal, a higher phase stability is necessary to reveal the detailed information in those regions. This is further exempli-

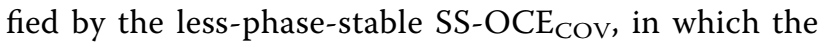
displacement boundary is more difficult to demarcate (Fig. 3a-d). Figure 3i, j shows the spatiotemporal images from SS-OCE $E_{\mathrm{COV}}$ and $\mathrm{SS}-\mathrm{OCE}_{\mathrm{CP}}$ at the depths indicated by the white arrows in Fig. 3a, e, respectively. The significantly reduced noise floor can be visualized

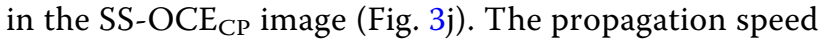
of the elasticity wave and Young's modulus of the phantom are shown in Fig. S1. 

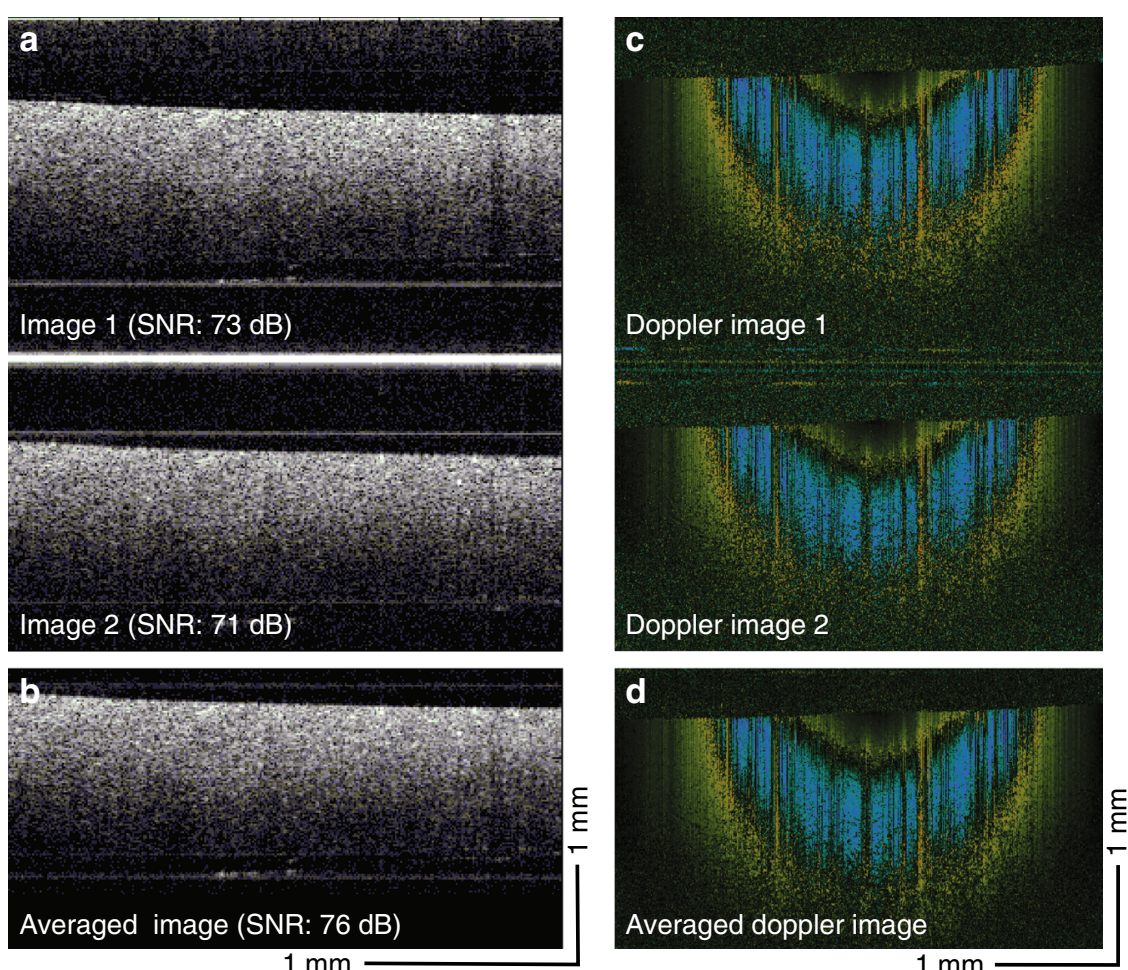

Fig. 2 SNR quantification. a, b Original and averaged OCT images, respectively. c, d Original and averaged Doppler OCT images, respectively
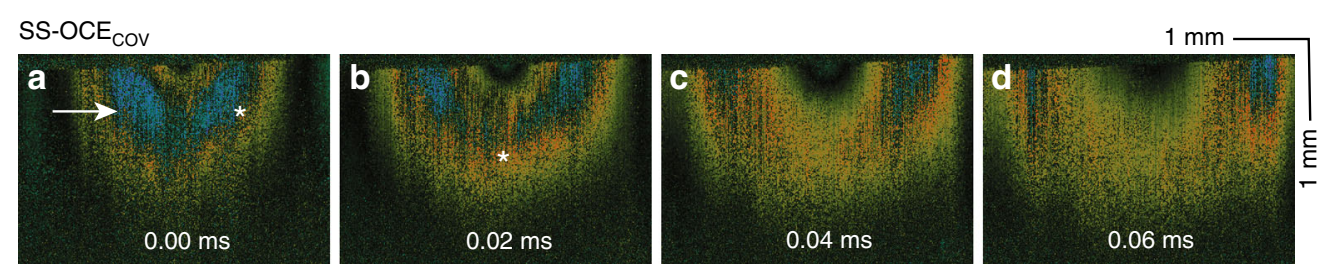

$$
\text { SS-OCE } \mathrm{CP}
$$
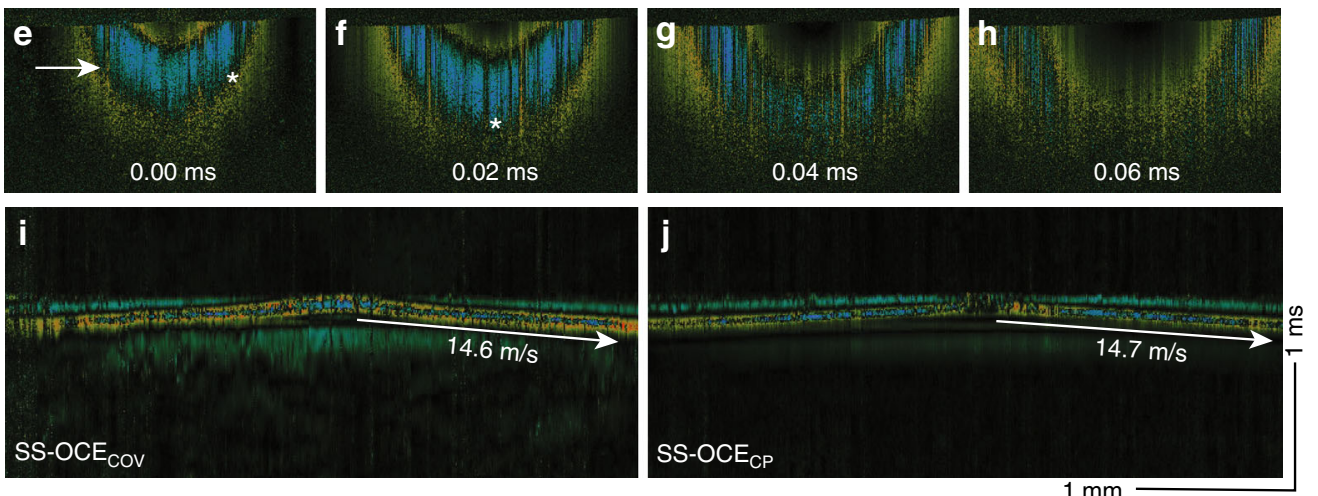

Fig. 3 Elastic wave in the silicone phantom. Time-lapse Doppler OCT B-scans obtained using (a-d) SS-OCE Spatiotemporal Doppler OCT at a depth indicated by the white arrows in (a) and (e), respectively

\section{In vivo rabbit experiment}

We further verified the performance of the proposed SS-OCE $E_{C P}$ in a rabbit model. Figure $4 a-d$ and Fig. $4 \mathrm{e}-\mathrm{h}$ show the time-lapse Doppler OCT B-scans of rabbit cornea obtained using SS-OCE $\mathrm{COV}_{\text {and }} \mathrm{SS}-\mathrm{OCE}_{\mathrm{CP}}$, respectively. In these figures, an elastic wave that 

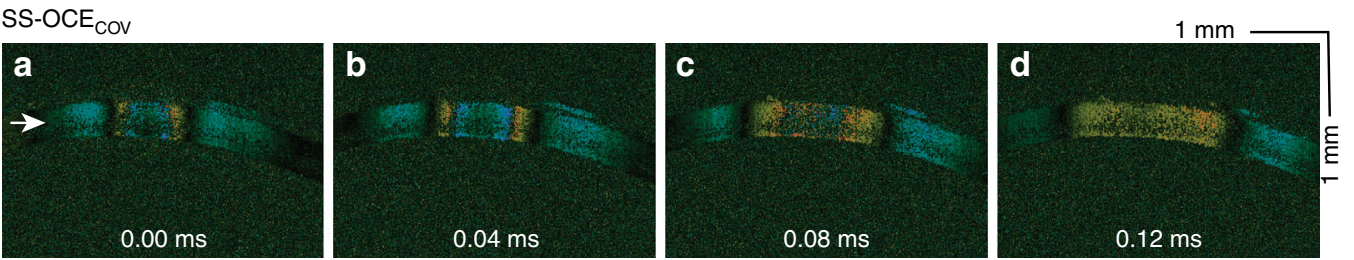

SS-OCE
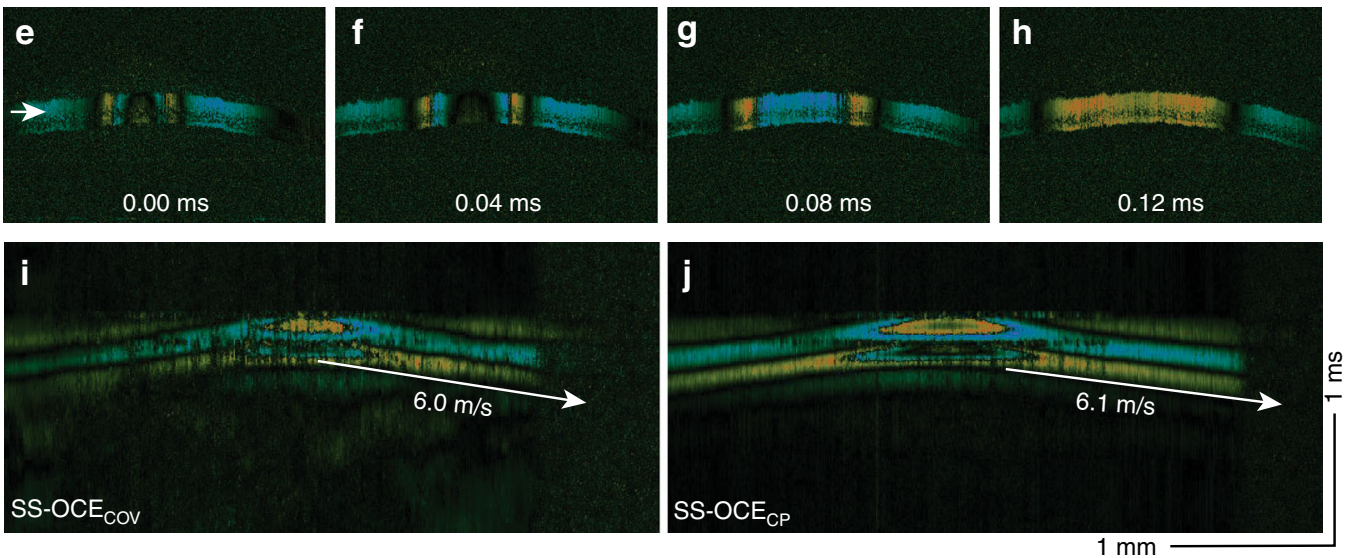

Fig. 4 Elastic wave in rabbit cornea. a-d Time-lapse Doppler OCT B-scans from the SS-OCECov system. e-h Time-lapse Doppler OCT B-scans from the common-path OCE system. i, j Spatiotemporal Doppler OCT at a depth indicated by the white arrows in (a) and (e), respectively

propagates outwards from the center can be visualized. Figure $4 \mathrm{i}, \mathrm{j}$ shows the corresponding spatiotemporal images and propagation speeds of two OCE systems at the depths indicated by the white arrows in Fig. 4a, e. The propagation speed of the elasticity wave and Young's modulus of the cornea are shown in Fig. S1. The results concur with the phantom experiment, demonstrating the enhanced phase stability of SS-OCE $\mathrm{CP}_{\mathrm{CP}}$, which allows for a more pronounced displacement boundary, an improved SNR in the deeper region, and reduced background noise.

A highly phase-stable OCE system can detect smaller displacements with improved accuracy. To investigate the influence of the generated ARF amplitude on the phase information retrieval, we applied three different levels of ARF, from 800 to $400 \mathrm{mV}$, using the same rabbit cornea model (Fig. 5). In all cases, SS-OCE $\mathrm{CP}_{\mathrm{C}}$ provided a better visualization of elastic wave propagation than did SS$\mathrm{OCE}_{\mathrm{COV}}$. In the $800-\mathrm{mV}$ SS-OCE $\mathrm{COV}$ case, the phase error can be observed at $0.12 \mathrm{~ms}$ in Fig. 5a. When

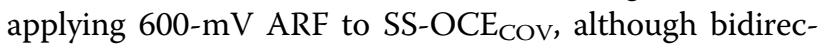
tional wave propagation can be identified in the center at $0 \mathrm{~ms}$ (Fig. 5d), only one direction of displacement was observed at $0.04 \mathrm{~ms}$ (Fig. 5c). A moderately poorer SNR was also observed in the $400-\mathrm{mV}$ SS-OCE $\mathrm{COV}$ (Figs. 5e versus $\mathrm{f}$ ). The corresponding spatiotemporal Doppler OCT images are shown in Fig. 6.

Last, we tested the change in elastic wave velocity in rabbit corneas in vivo with normal and high intraocular pressure (IOP) to further verify the capability of SS$\mathrm{OCE}_{\mathrm{CP}}$. A positive correlation between corneal elasticity and IOP was previously reported, and our results (Supplementary Material, Figs S2 and S3), demonstrating the same correlation, agree well with those of the previous study $^{37}$.

\section{Discussion}

OCE benefits from OCT, providing the ability to measure biological tissue with micrometer spatial resolution and subnanometre displacement sensitivity. The static operation principle of SD-OCT contributes to its wide use in OCE. However, recent advancements in SS-OCT have proven its utility in OCE, especially with the advantages of enhanced imaging range, depth, and speed, increased SNR, and reduced phase washout. The phase stability of SS-OCT can be improved through optical and electrical optimization, but reducing the phase fluctuation in a conventional SS-OCT setup is fundamentally challenging, as the reference and sample signals travel through different optical paths. We have previously demonstrated a method to optimize the electrical components in an SS-OCT system to achieve high-phase stability ${ }^{38}$, and in this study, we employed a common-path configuration to further attain the peak performance of SS-OCE. This was accomplished by enhancing the SNR through averaging, minimizing the timing jitter by adjusting the electrical delay, and reducing the fluctuation of the OPD via the 


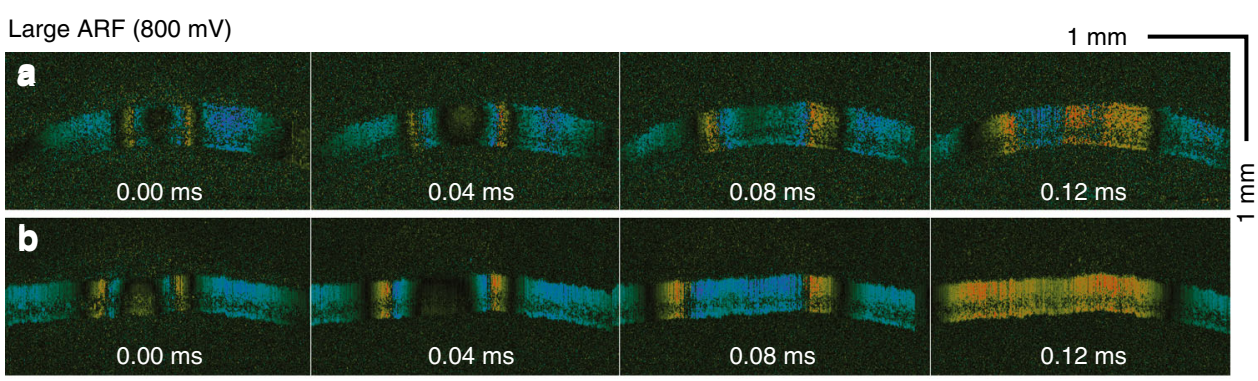

Medium ARF $(600 \mathrm{mV})$

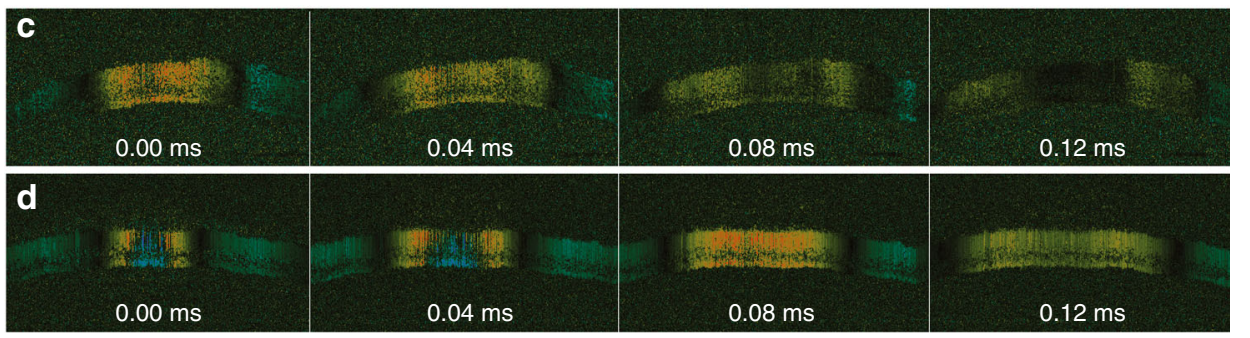

Small ARF $(400 \mathrm{mV})$
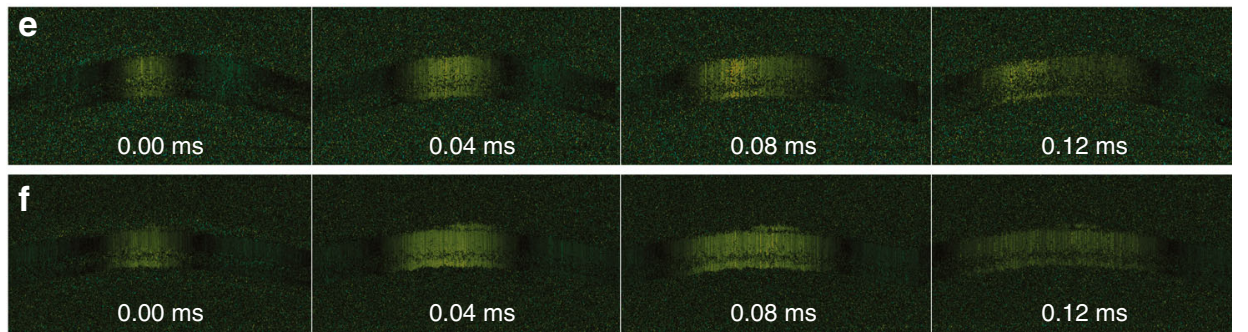

Fig. 5 Time-lapse Doppler OCT B-scans from SS-OCE ${ }_{\mathrm{COv}}$ and SS-OCE $\mathrm{CP}_{\text {s }}$ systems with different ARFs. a, c, e Time-lapse Doppler OCT B-scans from the SS-OCE $E_{C o v}$ system with large, medium, and small ARFs, respectively. b, d, f Time-lapse Doppler OCT B-scans from the SS-OCE large, medium, and small ARFs, respectively

common-path configuration. The resulting $\mathrm{SS}_{-} \mathrm{OCE}_{\mathrm{CP}}$ demonstrated a phase stability of $4.2 \mathrm{mrad}$, which was not only obtained in the system characterization but also achieved during the experiments. In addition to the 40fold improvement in the phase stability compared to that of SS-OCE $\mathrm{COV}_{\text {, }}$ SS-OCE $\mathrm{E}_{\mathrm{CP}}$ showed a 3-dB improvement in the imaging SNR, all without the need for external stabilization or extravagant post-processing.

The phase performance of our SS-OCE ${ }_{C P}$ was first validated using a tissue-mimicking silicone phantom. The improved phase stability was reflected by the more pronounced displacement boundary of the elastic wave than that of SS-OCE $\mathrm{COV}_{\mathrm{C}}$. We repeated the experiment using an in vivo rabbit corneal model to further demonstrate the improved capability of SS-OCE $\mathrm{E}_{\mathrm{CP}}$ to retrieve precise phase information for elasticity quantification. The results of the IOP experiment are also supported by reported studies ${ }^{37}$. Additionally, the improved displacement sensitivity provided by $S S-O C E_{C P}$ can reduce the minimum ARF required to induce a detectable tissue displacement, which is essential for future clinical translation.
Although we have demonstrated the superior performance of SS-OCE $\mathrm{CP}_{\mathrm{CP}}$, there are a few design improvements that can be implemented. First, in the proposed optical configuration, the reference signals are generated by the front and back surfaces of the 3-mm wedged window. To achieve the optimal SNR, the travel distance of the detection beam from the scan lens to the first surface of the window must be constant to provide a uniform backreflected signal; even a slight deviation will cause a variation in the OPD and back-reflected power during scanning, resulting in a reduced SNR. For applications that require scanning of a larger area, a scan lens that provides a larger field of view should be used. Furthermore, for three-dimensional OCE, a $4 \mathrm{~F}$ optical corrector can be incorporated into the scanning design to ensure a constant entrance pupil and OPD.

In addition, we previously demonstrated a confocal alignment between the OCT detection beam and the excitation via a coaxial configuration for high excitation efficiency $^{15,32}$. A confocal/coaxial setup can improve the ease of use and reduce the form factor of the sample arm, 


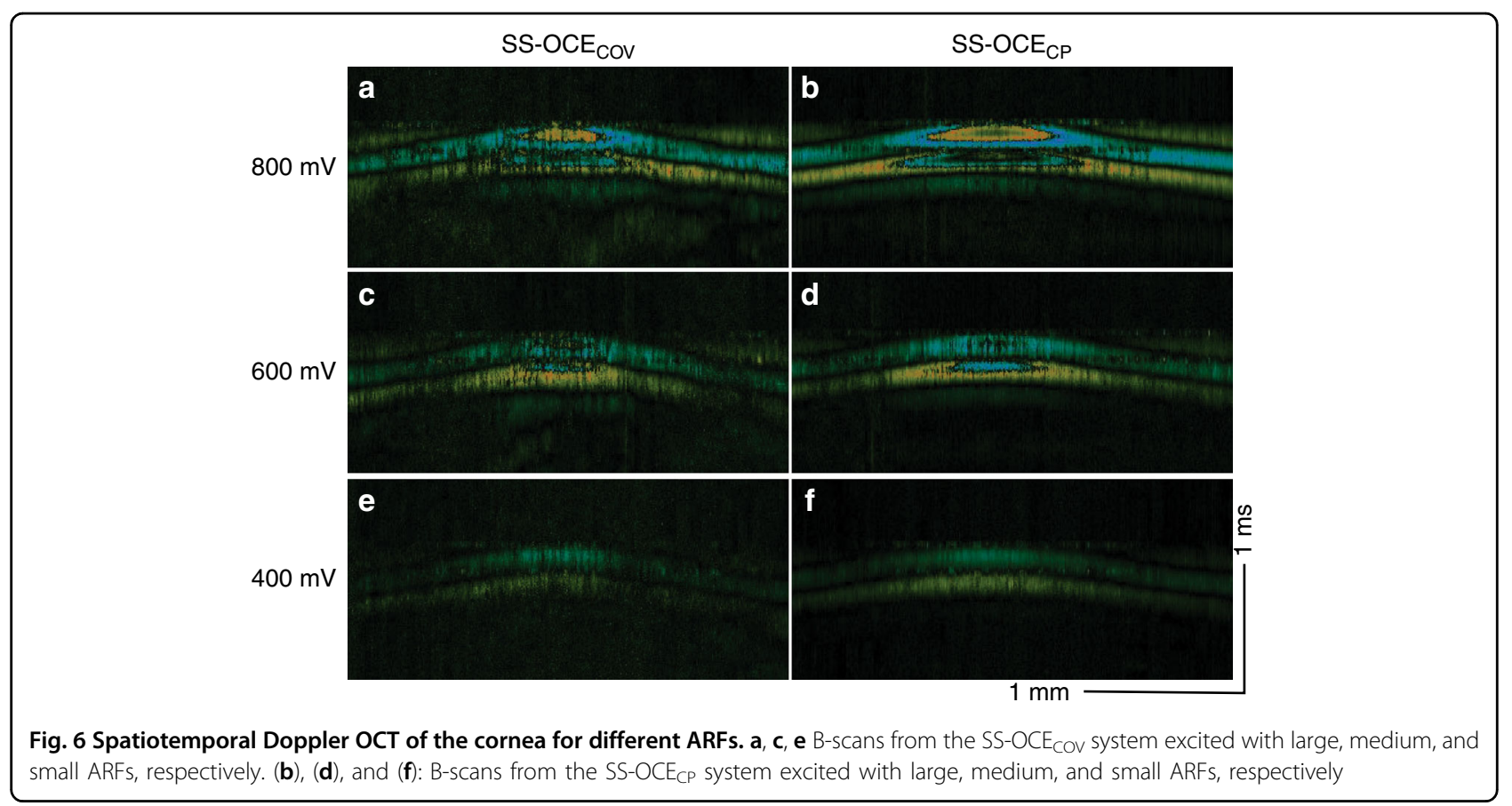

further facilitating the translation of this technology to clinical use. In our current SS-OCE $\mathrm{E}_{\mathrm{CP}}$ design, the wedged window prevents this confocal setup, as the ARF will be attenuated by the window. Alternatively, the wedged window can be fabricated using an optically and acoustically transparent material, such as Pebax $^{\circledR}$ and lowdensity polyethylene $\mathrm{e}^{39,40}$. With this window, a ring-shaped ultrasound transducer can be inserted between the scan lens and the window for excitation, allowing the OCT detection beam to travel through the center of the transducer for imaging.

Finally, the light source of the SS-OCE $\mathrm{CP}_{\mathrm{CP}}$ is a verticalcavity surface-emitting laser with an internal MZI that provides a k-clock signal at $\sim 400 \mathrm{MHz}$, allowing for an imaging range of $\sim 11 \mathrm{~mm}$ in standard OCT or $\sim 5.5 \mathrm{~mm}$ when utilizing the proposed averaging method that enhances the SNR by $3 \mathrm{~dB}$. While a $5.5-\mathrm{mm}$ imaging range may be sufficient in many applications, a long imaging range can be achieved through doubling the $\mathrm{k}$ clock frequency by building an external circuit or through the dual edge sampling provided by certain waveform digitizers. A custom-built external k-clock can also be considered. Alternatively, an acousto-optic modulator can be incorporated to shift the frequency of the interferometer signal to take advantage of the full bandwidth of the frequency ${ }^{41}$. With respect to achieving a better penetration depth, swept-source lasers with a longer center wavelength, such as $1.7 \mu \mathrm{m}$, can be utilized $^{42}$.

In summary, we have reported the first OCE system based on SS-OCT with a common-path configuration. A phase stability of $4.2 \mathrm{mrad}$ was obtained, and the feasibility and performance of our SS-OCE $\mathrm{CP}_{\mathrm{CP}}$ were tested and validated using a phantom and in vivo rabbit model. This highly phase-stable system can quantify displacement in the subnanometre range, and we believe that it has great potential in other applications that require precision phase measurement, such as flowmetry ${ }^{11}$, vibrometry ${ }^{43}$, and molecular imaging ${ }^{44}$.

\section{Materials and methods System setup}

An SS-OCE $E_{C P}$ system was designed and constructed. The swept laser (SL1310V1-10048, Thorlabs, Inc., NJ) has a repetition rate of $100 \mathrm{kHz}$, a center wavelength of $1310 \mathrm{~nm}$, and a bandwidth of $100 \mathrm{~nm}$. The system has an imaging range of $11 \mathrm{~mm}$, which makes the generation of two OCT images in different frequency domains possible. The output light from the laser source is split by a $90: 10$ optical fiber coupler, with $90 \%$ of the light propagating to the sample through a circulator, a collimator, an objective scan lens (LSM04, Thorlabs, Inc., NJ), and a wedged window, as shown in Fig. 7. The common-path configuration is achieved through the 30 -arcmin wedged window. In contrast to a flat window, the 30 -arcmin angle on the front surface effectively reduces the autocorrelation fringe patterns generated within the window cavity, as shown in Fig. 8. In this setup, two interfaces exist: the airglass front surface and the glass-gel back surface. The indices of refraction of the air, glass (the wedged window), and ultrasound gel are 1.0, 1.5, and 1.34, respectively. The back surface of the wedged window is perpendicular to 


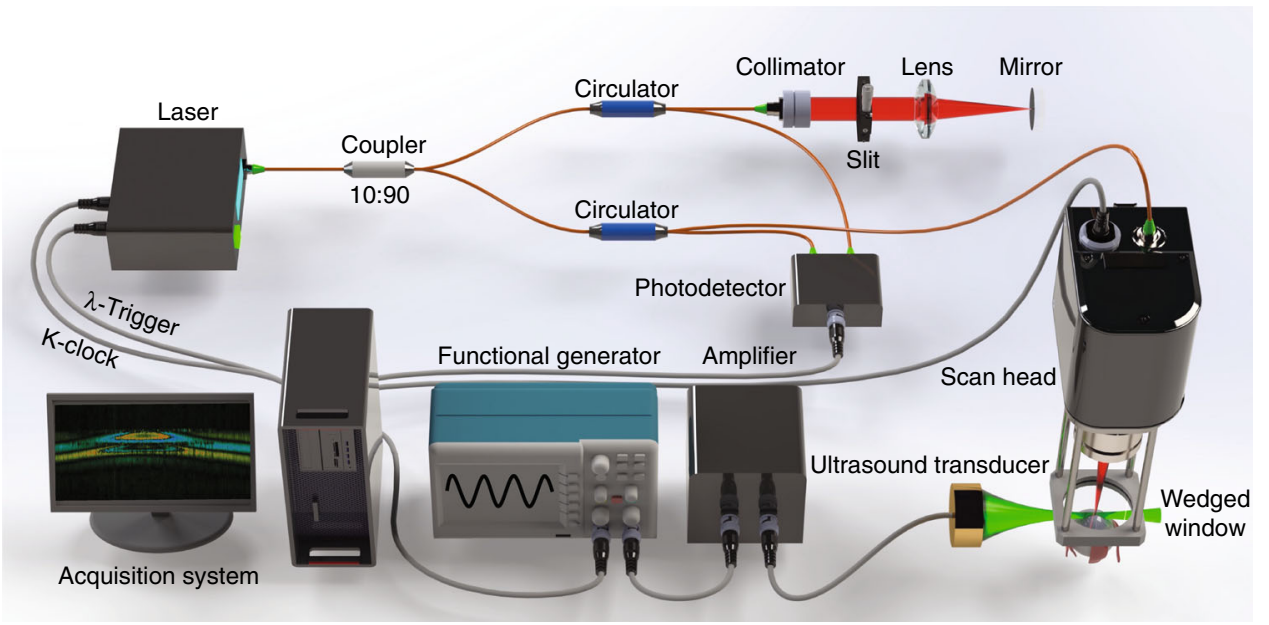

Fig. 7 Schematics of the SS-OCEEP system

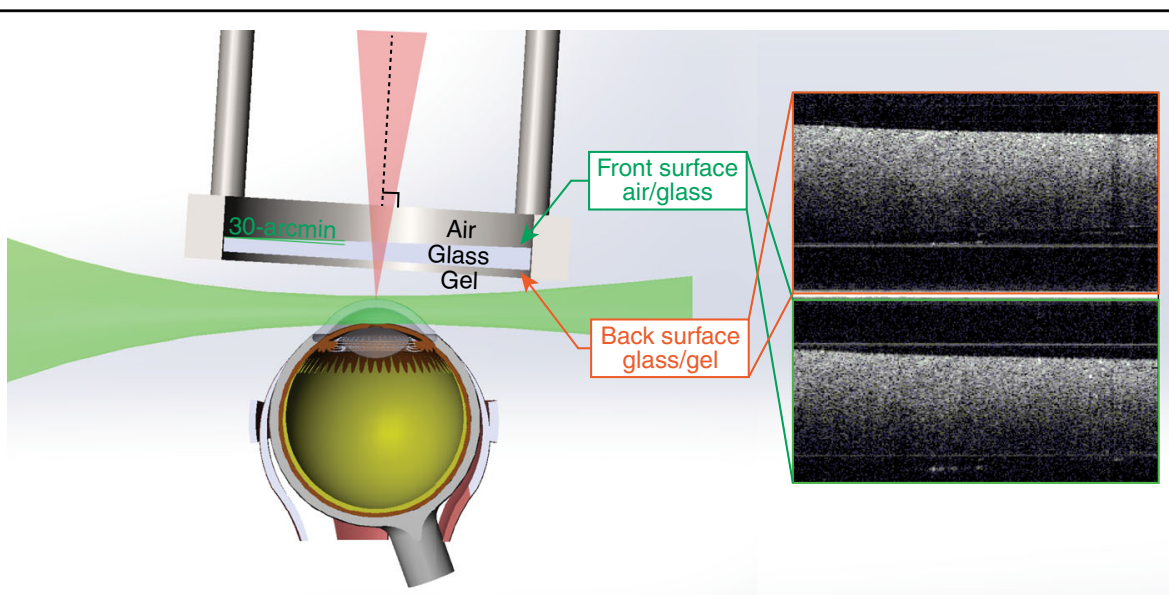

Fig. 8 Schematics of the 30-arcmin wedged window

the OCT scanning beam, while the front surface is shifted by 30 arcmin from the normal direction. The differences in the refractive indices and in the corresponding incident angles contribute to the discrepancy in reflectance of the two surfaces, but this can be compensated for by adjusting the position of the wedged window relative to the scan lens because the collected power of the focusing backreflected light is spatially dependent (Supplementary Material, Fig. S4). As such, two back-reflected reference signals of similar power interfere with the backscattered sample signal to generate two distinct fringes corresponding to the top and bottom images. The two interferences are then delivered to one channel of the balanced photodetector. The wedged window has a thickness of $3 \mathrm{~mm}$, which corresponds to a 3.4-mm axial separation of the generated OCT images calculated based on the ultrasound gel reflective index of 1.34. Additionally, the two images can be averaged to enhance the SNR and minimize speckles. To enable balanced detection, the remaining $10 \%$ of the light propagates through a compensation arm consisting of a circulator, a collimator, an adjustable slit and a mirror. The back-reflected light from the compensation arm is detected by the second channel of the balanced photodetector to offset the DC component of the generated interference fringes. A balanced photodetector with a bandwidth from $30 \mathrm{kHz}$ to $1.6 \mathrm{GHz}$ was selected to minimize timing jitters to enhance the phase stability and to enable a long imaging range for retrieving the two OCT interference fringes from different frequency domains ${ }^{14}$.

For the displacement excitation, a custom-built $4.5-\mathrm{MHz}$ ultrasound transducer with a focal length of $35 \mathrm{~mm}$ is placed approximately orthogonal to the scan lens. A function generator is synchronized with the 
$\lambda$-trigger signal to generate a $4.5-\mathrm{MHz}$ sine wave (duration: $200 \mu \mathrm{s}$ ) that is then amplified by approximately $42 \mathrm{~dB}$ to drive the ultrasound transducer for tissue excitation. The space between the wedged window, the ultrasound, and the sample is filled with ultrasound gel to couple the ARF.

For comparison, $\mathrm{SS}-\mathrm{OCE}_{\mathrm{CP}}$ can be quickly converted to SS-OCE $\mathrm{COV}_{\mathrm{C}}$ during the experiments while maintaining the exact location of the sample and the ultrasound transducer relative to the OCT detection beam (Supplementary Material, Fig. S5).

\section{Data acquisition, synchronization, and signal processing}

Two timing signals produced by the swept-source laser are utilized for data acquisition and synchronization. The k-clock signal generated by the internal MZI provides a timing of equal wavenumber spacings, and the $\lambda$-trigger signal is produced by the internal FBG to give a temporal mark for each wavelength sweeping. In conventional SSOCT or SS-OCE imaging systems, a series of OCT signal points is digitized starting at the edge of the $\lambda$-trigger, while the signal digitization is clocked by the k-clock signal for k-linear signal sampling. However, in this mode of operation, random timing errors of the signal edges can be magnified to significant timing fluctuations between the $\mathrm{k}$-clock and $\lambda$-trigger through a process termed edge collision $^{38}$. To achieve the best stability, the signal delays are adjusted to the optimal values for the k-clock, $\lambda$ trigger and OCE signals. A previous study on the phase stability with the same type of swept lasers suggested that a very high stability could be obtained using this method $^{38}$.

The propagation velocity of the elastic wave provides a direct measurement of the biomechanical property, as pre-calibration is not necessary to convert the displacement to elasticity. To visualize the elastic wave propagation, an M-B scanning protocol is utilized to induce and detect the displacement. At each lateral position, 500 Alines are acquired to record the phase change over time (M-mode). The ultrasound transducer is excited after a trigger delay of $1 \mathrm{~ms}$ to generate an ARF with a duration of $200 \mu \mathrm{s}$ for each M-mode acquisition. After one Mmode acquisition, the galvanometer scanner moves the detection beam to the next lateral position, and the same step is repeated. A total of $3000 \mathrm{M}$-mode images are acquired for each dataset to convert to B-mode images. The scanning protocol is summarized in Fig. S6 in the Supplementary Material. The phase-resolved Doppler algorithm is applied to extract the temporal phase information. With a time interval of $50 \mu \mathrm{s}$, inter-A-line analysis is performed to obtain time-lapse Doppler OCT B-scans and spatiotemporal Doppler OCT images. Young's modulus can then be calculated by determining the propagation velocity using the spatiotemporal Doppler images.
Because the different boundary conditions yield different propagation modes of the elastic waves, a specific equation is used to calculate the elasticity based on the sample types $^{8,45}$. In our experiment, Young's modulus, $E$, is calculated based on the Rayleigh wave velocity, $V_{\mathrm{R}}$, and Lamb wave velocity, $V_{\mathrm{L}}$, for the tissue-mimicking phantom and rabbit cornea, respectively. The data processing steps and key equations for elasticity calculation are detailed in Fig. S7 in the Supplementary Material.

\section{Phantom preparation}

To fabricate the silicone-based phantom that mimics tissue biomechanical properties, $2 \mathrm{~g}$ of titanium dioxide was added to every $100 \mathrm{~g}$ of silicone rubber base (P4-Part B, Eager Polymers, Inc., IL) and mixed using an ultrasonic cleaner. Then, 1 part of the silicone rubber base with wellmixed titanium dioxide was added to 16 parts of silicone activator (P4-Part A, Eager Polymers, Inc., IL). After the base was gently mixed with the activator using a stirring rod, the mixture was placed into a vacuum chamber until all the air bubbles trapped inside the mixture were removed. The mixture was then poured into a container for molding and cured for $24 \mathrm{~h}$. The final phantom had dimensions of $40 \mathrm{~mm} \times 40 \mathrm{~mm} \times 10 \mathrm{~mm}$.

\section{In vivo rabbit experiment preparation}

To induce the initial anesthesia, the rabbit (New Zealand white rabbit, male, $\sim 3 \mathrm{~kg}$ ) was administered a ketamine-xylazine mixture $(35 \mathrm{mg} / \mathrm{kg}$ and $5 \mathrm{mg} / \mathrm{kg}$, respectively) subcutaneously. Two drops of $2.5 \%$ proparacaine hydrochloride were applied topically for local anesthesia. After conforming to the proper depth of anesthesia, the rabbit was placed onto a stage for position adjustment. The rabbit's eye was covered with sterile ultrasound gel to provide a conductive medium between the eye and the acoustic wave for the IOP experiment. To obtain a higher IOP, the rabbit eye was carefully proptosed, and a sterile latex drape with an aperture was put through the globe to maintain proptosis. Eye proptosis in rabbits typically increases the IOP to $\sim 50 \mathrm{mmHg}$. All procedures were reviewed and approved by the Institutional Animal Care and Use Committee at the University of California, Irvine, under protocol \#AUP-19-042.

\section{Acknowledgements}

National Institutes of Health (R01EY-026091, R01EY-028662, R01HL-125084, R01HL-127271), American Heart Association (18PRE34050021), and the National Science Foundation (DGE-1839285).

\footnotetext{
Author details

${ }^{1}$ Beckman Laser Institute, University of California, Irvine, Irvine, CA 92612, USA. ${ }^{2}$ Department of Biomedical Engineering, University of California, Irvine, Irvine, CA 92617, USA. 'Department of Physics, Kookmin University, Seoul 02707, South Korea
} 


\section{Author contributions}

L.Y. conceived the idea, designed the system setup, conducted the animal experiments, analyzed the data, and wrote the manuscript. M.S. analyzed the data and edited the manuscript. C.J. edited the manuscript and conducted the animal experiments. Z.Z. drew the system diagrams. C.Z. conceived the idea, supervised the research, and edited the manuscript. All authors discussed and contributed to the paper.

\section{Conflict of interest}

Dr. Chen has a financial interest in OCT Medical Imaging, Inc., which, however, did not support this work.

Supplementary information is available for this paper at https://doi.org/ 10.1038/s41377-020-0297-9.

Received: 29 October 2019 Revised: 16 March 2020 Accepted: 19 March 2020

Published online: 13 April 2020

\section{References}

1. Schmitt, J. M. OCT elastography: imaging microscopic deformation and strain of tissue. Opt. Express 3, 199-211 (1998).

2. Kennedy, B. F., Wijesinghe, P. \& Sampson, D. D. The emergence of optical elastography in biomedicine. Nat. Photonics 11, 215-221 (2017).

3. Feng, $Y$. et al. Viscoelastic properties of the ferret brain measured in vivo at multiple frequencies by magnetic resonance elastography. J. Biomech. $\mathbf{4 6}$ 863-870 (2013).

4. Zhang, X. Y. et al. Noninvasive assessment of age-related stiffness of crystalline lenses in a rabbit model using ultrasound elastography. Biomed. Eng. OnLine 17, 75 (2018)

5. Yun, S. H. \& Chernyak, D. Brillouin microscopy: assessing ocular tissue biomechanics. Curr. Opin. Ophthalmol. 29, 299-305 (2018).

6. Muthupillai, R. \& Ehman, R. L. Magnetic resonance elastography. Nat. Med. 2, 601-603 (1996).

7. Qu, Y. Q. et al. Acoustic radiation force optical coherence elastography of corneal tissue. IEEE J. Sel. Top. Quantum Electron. 22, 6803507 (2016).

8. Kirby, M. A. et al. Optical coherence elastography in ophthalmology. J. Biomed. Opt. 22, 121720 (2017).

9. Qu, Y. Q. et al. Miniature probe for mapping mechanical properties of vascular lesions using acoustic radiation force optical coherence elastography. Sci. Rep. 7, 4731 (2017).

10. Song, S. Z. et al. Strategies to improve phase-stability of ultrafast swept source optical coherence tomography for single shot imaging of transient mechanical waves at $16 \mathrm{kHz}$ frame rate. Appl. Phys. Lett. 108, 191104 (2016).

11. Zhao, Y. H. et al. Phase-resolved optical coherence tomography and optical Doppler tomography for imaging blood flow in human skin with fast scanning speed and high velocity sensitivity. Opt. Lett. 25, 114-116 (2000).

12. Szkulmowska, A. et al. Phase-resolved Doppler optical coherence tomography-limitations and improvements. Opt. Lett. 33, 1425-1427 (2008).

13. Qu, Y. Q. et al. In vivo elasticity mapping of posterior ocular layers using acoustic radiation force optical coherence elastography. Investigative Ophthalmol. Vis. Sci. 59, 455-461 (2018).

14. Li, Y. et al. Simultaneously imaging and quantifying in vivo mechanical properties of crystalline lens and cornea using optical coherence elastography with acoustic radiation force excitation. APL Photonics 4, 106104 (2019).

15. He, Y. M. et al. Confocal shear wave acoustic radiation force optical coherence elastography for imaging and quantification of the in vivo posterior eye. IEEE J. Sel. Top. Quantum Electron. 25, 7200107 (2019).

16. Kang, J. U. et al. Common-path optical coherence tomography for biomedical imaging and sensing. J. Optical Soc. Korea 14, 1-13 (2010).

17. Vakhtin, A. B. et al. Common-path interferometer for frequency-domain optical coherence tomography. Appl. Opt. 42, 6953-6958 (2003).

18. Tan, K. M. et al. In-fiber common-path optical coherence tomography using a conical-tip fiber. Opt. Express 17, 2375-2384 (2009).

19. Lan, G. P. et al. Common-path phase-sensitive optical coherence tomography provides enhanced phase stability and detection sensitivity for dynamic elastography. Biomed. Opt. Express 8, 5253-5266 (2017).

20. Leartprapun, N. et al. Photonic force optical coherence elastography for threedimensional mechanical microscopy. Nat. Commun. 9, 2079 (2018).
21. Ahmad, A. et al. Magnetomotive optical coherence elastography using magnetic particles to induce mechanical waves. Biomed. Opt. Express 5, 2349-2361 (2014).

22. Zhang, H. Q. et al. Optical coherence elastography of cold cataract in porcine lens. J. Biomed. Opt. 24, 036004 (2019).

23. Qu, Y. Q. et al. Quantified elasticity mapping of retinal layers using synchronized acoustic radiation force optical coherence elastography. Biomed. Opt. Express 9, 4054-4063 (2018).

24. He, Y. M. et al. Characterization of oviduct ciliary beat frequency using real time phase resolved Doppler spectrally encoded interferometric microscopy. Biomed. Opt. Express 10, 5650-5659 (2019).

25. Hendargo, H. C. et al. Doppler velocity detection limitations in spectrometerbased versus swept-source optical coherence tomography. Biomed. Opt. Express 2, 2175-2188 (2011).

26. De Boer, J. F., Leitgeb, R. \& Wojtkowski, M. Twenty-five years of optical coherence tomography: the paradigm shift in sensitivity and speed provided by Fourier domain OCT [Invited]. Biomed. Opt. Express 8, 3248-3280 (2017).

27. Song, S. Z., Xu, J. J. \& Wang, R. K. Long-range and wide field of view optical coherence tomography for in vivo 3D imaging of large volume object based on akinetic programmable swept source. Biomed. Opt. Express 7, 4734-4748 (2016).

28. Tsai, M. T. et al. Quantitative phase imaging with swept-source optical coherence tomography for optical measurement of nanostructures. IEEE Photonics Technol. Lett. 24, 640-642 (2012).

29. De Stefano, V. S. et al. Live human assessment of depth-dependent corneal displacements with swept-source optical coherence elastography. PLOS ONE 13, e0209480 (2018).

30. Razani, M. et al. Feasibility of optical coherence elastography measurements of shear wave propagation in homogeneous tissue equivalent phantoms. Biomed. Opt. Express 3, 972-980 (2012).

31. Manapuram, R. K. et al. In vivo estimation of elastic wave parameters using phase-stabilized swept source optical coherence elastography. J. Biomed. Opt. 17, 100501 (2012)

32. Zhu, J. et al. Coaxial excitation longitudinal shear wave measurement for quantitative elasticity assessment using phase-resolved optical coherence elastography. Opt. Lett. 43, 2388-2391 (2018).

33. Singh, $M$. et al. Evaluating the effects of Riboflavin/UV-A and Rose-Bengal/ Green light cross-linking of the rabbit cornea by noncontact optical coherence elastography. Investigative Ophthalmol. Vis. Sci. 57, OCT112-OCT120 (2016).

34. Zhu, J. et al. Imaging and characterizing shear wave and shear modulus under orthogonal acoustic radiation force excitation using OCT doppler variance method. Opt. Lett. 40, 2099-2102 (2015).

35. Zhi, Z. W. et al. 4D optical coherence tomography-based micro-angiography achieved by 1.6-MHz FDML swept source. Opt. Lett. 40, 1779-1782 (2015).

36. Zhang, J. et al. High-dynamic-range quantitative phase imaging with spectral domain phase microscopy. Opt. Lett. 34, 3442-3444 (2009).

37. Ambroziński, Ł. et al. Acoustic micro-tapping for non-contact 4D imaging of tissue elasticity. Sci. Rep. 6, 38967 (2016).

38. Moon, S. \& Chen, Z. P. Phase-stability optimization of swept-source optical coherence tomography. Biomed. Opt. Express 9, 5280-5295 (2018).

39. Yang, J. M. et al. Photoacoustic endoscopy. Opt. Lett. 34, 1591-1593 (2009).

40. Li, Y. et al. PMN-PT/Epoxy 1-3 composite based ultrasonic transducer for dualmodality photoacoustic and ultrasound endoscopy. Photoacoustics 15, 100138 (2019).

41. Jing, J. et al. High-speed upper-airway imaging using full-range optical coherence tomography. J. Biomed. Opt. 17, 110507 (2012).

42. Li, Y. et al. Intravascular optical coherence tomography for characterization of atherosclerosis with a 1.7 micron swept-source laser. Sci. Rep. 7, 14525 (2017).

43. Choi, S. et al. Multifrequency-swept optical coherence microscopy for highspeed full-field tomographic vibrometry in biological tissues. Biomed. Opt Express 8, 608-621 (2017).

44. Makita, S. \& Yasuno, Y. Detection of local tissue alteration during retinal laser photocoagulation of ex vivo porcine eyes using phase-resolved optical coherence tomography. Biomed. Opt. Express 8, 3067-3080 (2017).

45. Zhu, J., He, X. D. \& Chen, Z. P. Perspective: current challenges and solutions of Doppler optical coherence tomography and angiography for neuroimaging APL Photonics 3, 120902 (2018). 